\title{
Microwave-to-terahertz dielectric resonators for liquid sensing in microfluidic systems
}

\author{
N. Klein ${ }^{\mathrm{a}}$, C. Watts ${ }^{\mathrm{a}}$, S. M. Hanham ${ }^{\mathrm{a}}$, W. J. Otter ${ }^{\mathrm{b}}$, M.M. Ahmad ${ }^{\mathrm{b}}$ and S. Lucyszyn ${ }^{\mathrm{b}}$ \\ ${ }^{a}$ Department of Materials, ${ }^{b}$ Department of Electrical and Electronic Engineering \\ Imperial College London, South Kensington Campus, London SW7 2AZ, United Kingdom
}

\begin{abstract}
The microwave-to-terahertz frequency range offers unique opportunities for the sensing of liquids based on the degree of molecular orientational and electronic polarization, Debye relaxation due to intermolecular forces between (semi-)polar molecules and collective vibrational modes within complex molecules. Methods for the fast dielectric characterization of (sub-)nanolitre volumes of mostly aqueous liquids and biological cell suspensions are discussed, with emphasis on labon-chip approaches aimed towards single-cell detection and label-free flow cytometry at microwave-to-terahertz frequencies. Among the most promising approaches, photonic crystal defect cavities made from high-resistivity silicon are compared with metallic split-ring resonant systems and high quality factor (Q-factor) whispering gallery-type resonances in dielectric resonators. Applications range from accurate haemoglobin measurements on nanolitre samples to label-free detection of circulating tumor cells.
\end{abstract}

Keywords: microwave and terahertz liquid characterization, label free cancer cell detection, dielectric resonators, photonic crystal resonators

\section{INTRODUCTION}

An important goal of medical diagnostics is the early state detection and monitoring of cancer through the analysis of body liquids including urine, saliva and less easily-accessible liquids like the pancreatic fluid. Most of the existing diagnostic approaches rely on biochemical detection based on tumor markers, but quite often the tumor markers do not express [1]. This leads to a large uncertainty of early-state cancer detection based on blood analysis. Therefore, highly invasive biopsy methods still represent the gold standard, although it is well known that circulating tumor cells (CTCs) theoretically provide evidence for cancer and even detailed information about the type of cancer [2]. Therefore, label free detection and separation of tumor cells within their natural liquid environment, in particular CTCs, is the holy grail of cancer diagnostics. Among the possible physical properties which enable distinguishing cancer cells from healthy cells, the response of a cell to electromagnetic (EM) waves in principle enables fast detection and separation even in the case of extremely small numbers like CTCs. Optical scattering represents the most commonly used method to determine cell size and cell inhomogeneity by scattering within standard flow cytometry [3]. There is a natural demand to utilize lower frequency bands, and the maturing of microfluidic technology encourages the development and exploration of integrated microfluidic devices with EM detection capability over a wider range of frequencies.

The microwave-to-terahertz range is quite unique for this purpose because of fundamental physical facts: the cell membrane relaxation at $\mathrm{MHz}$ frequencies makes a cell quite transparent for frequencies above $1-5 \mathrm{GHz}$, which enables the determination of the average complex permittivity within an effective medium approach [4]. At the same time, the wavelength is still large in comparison to the cell size of 1-30 microns, which diminishes scattering effects and enables absorption measurements. Lastly, the high absorption by water - the main constituent of most cells - at microwave-toterahertz frequencies and its subtle modifications by proteins and surface water [5] enables highly sensitive detection. As such, it provides a complementary view of a given cell to existing techniques, and may become a key element for label free diagnosis within a microfluidic device with the strong potential to attain high throughput like in optical flow cytometry.

Terahertz Emitters, Receivers, and Applications VII, edited by Manijeh Razeghi, Alexei N. Baranov, John M. Zavada, Dimitris Pavlidis, Proc. of SPIE Vol. 9934, 99340F · (C) 2016 SPIE CCC code: $0277-786 \mathrm{X} / 16 / \$ 18 \cdot$ doi: $10.1117 / 12.2238545$ 


\subsection{Resonator methods and figure of merit}

EM resonator based measurements represent a powerful tool for achieving the highest possible sensitivity for the characterization of small volumes of liquids whose dimensions are comparatively small compared to the relatively long wavelength of microwave and terahertz radiation. A high Q-factor and a strong confinement of the electric field within the small volume of a microfluidic reservoir containing the liquid under test are the key requirements, but quite often a reduction of the resonator volume decreases the Q-factor. Cavity perturbation theory [6] can be employed to define a relevant figure of merit, such as the resonant frequency change $\Delta f$ normalized to the resonant half-width $\Delta f_{1 / 2}$ with the liquid in place for a given volume $V_{\mathrm{l}}$, say $1 \mathrm{nl}$. This can be expressed as

$$
\frac{\Delta f}{\Delta f_{1 / 2}}=\frac{\Delta f}{f_{r}} Q_{l} \approx \frac{Q_{l} \varepsilon^{\prime} \int_{\Delta V} E^{2} d V}{4 W} \approx \frac{Q_{l}}{4} \frac{\varepsilon^{\prime} E_{l}^{2} V_{l}}{W}
$$

with $Q_{1}$ denoting the loaded Q-factor with the liquid in place, $E_{1}$ the average electric field within the microfluidic reservoir, $W$ the stored energy of the resonant mode and $\varepsilon$ ' the real part of the dielectric permittivity. $E_{1}$ and $W$ can be easily extracted from an eigenmode analysis using EM field simulations, for example using CST Microwave Studio [7]. However, eigenmode solvers often have difficulties with lossy dielectrics, therefore Eq. 1 gives just the order of magnitude of the sensitivity. The large imaginary part $\varepsilon$ " of the complex permittivity $\varepsilon^{*}=\varepsilon^{\prime}+j \varepsilon$ "' of aqueous liquids over the entire microwave-to-terahertz range imposes limits on standard cavity perturbation theory: the normalized frequency shift according to Eq. 1 and the normalized shift of inverse quality factor $Q_{1} \Delta Q_{1}^{-1}$ (= liquid induced change of resonant half-width normalized to the half-width with the liquid in place) can be written as a complex function $\Omega$ of the complex permittivity, which in most cases cannot be easily separated into real and imaginary parts, as suggested by standard cavity perturbation theory.

$$
\Omega\left(\varepsilon^{*}\right) \equiv \frac{\Delta f}{\Delta f_{1 / 2}}\left(\varepsilon^{*}\right)+j Q_{l} \Delta Q_{l}^{-1}\left(\varepsilon^{*}\right) \neq \frac{\Delta f}{\Delta f_{1 / 2}}\left(\varepsilon^{\prime}\right)+j Q_{l} \Delta Q_{l}^{-1}\left(\varepsilon^{\prime \prime}\right)
$$

In spite of this peculiarity which adds some difficulties for performing quantitative measurements of the complex permittivity of aqueous liquids in microfluidic reservoirs by cavity perturbation techniques, Eq. 1 allows us to define the key design criteria for achieving high measurement sensitivity for small liquid volumes: high Q-factor, strong electric field enhancement within the microfluidic reservoir and small resonator volume i.e. small value of the total energy $W$. It is worth noting that for commercial eigenmode solvers the energy $W$ is often fixed (typically 1 Joule), which makes it easy to optimize a given structure by aiming for a high electric field strength $E_{1}$ within the microfluidic reservoir and the highest Q-factor.

\section{2: Dielectric resonators vs metallic resonators}

Planar resonators, multiple resonator filter structures and even metamaterials are attractive because of their high electric fields within capacitive gaps, like in coplanar waveguides, lumped element LC resonators or split ring resonators $[8,9]$. Microfluidic fabrication technology enables the integration of metal electrodes within microfluidic channels. However, the achievable Q-factors of planar resonators are fairly low, in particular when the microfluidic channels are filled with an aqueous liquid. Since the loss tangent of water between $10 \mathrm{GHz}$ and $1 \mathrm{THz}$ is about unity, a nearly complete filling of the capacitive gap by the liquid kills the resonance. A lower filling factor may be a bad compromise with respect to sensitivity. A possible solution to overcome this difficulty is discussed in Section 2.3. For frequencies above about $10 \mathrm{GHz}$ dielectric resonators represent a suitable choice. In particular, whispering gallery (WG) type modes in cylindrical dielectric discs, which are hybrid modes (HE or EH, i.e. with a dominant axial magnetic or electric field component, respectively) with high (>6) azimuthal mode number, allow for very high Q-factors which are only limited by losses in the dielectric material [10]. Fig. 1 from our previous work [4] shows a whispering gallery mode resonator of low azimuthal index (6) embedded in a semi-open metal shielded cavity. The liquid under test flows through a linear channel which intersects the field maxima of the WG resonance in the ceramic dielectric disc placed underneath the plastic microfluidic chip. The system allows dielectric measurements on samples of about 1 microlitre volume at typical 
Q-factors of 4,000, which allows highly accurate measurements of small changes of the complex permittivity of the liquid. The microwave ceramics have the additional advantage of a nearly zero temperature dependence of the resonant frequency. As an example, accurate haemoglobin concentration measurements have been demonstrated on blood samples, from differential resonant frequency measurements of blood serum vs serum plus added red blood cells. It is important to note that the membranes of the red blood cells are transparent at the given frequency of approximately $10 \mathrm{GHz}$. Due to the low permittivity of haemoglobin - in comparison to water - the measured resonant frequency exhibits a linear dependence on the haemoglobin concentration in water, which enables an easy calibration of the system.
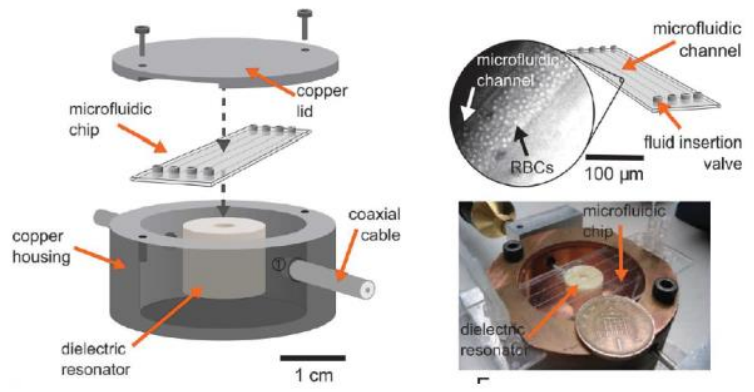

Fig. 1: Ceramic $10 \mathrm{GHz}$ whispering gallery type resonator for the measurement of haemoglobin $(\mathrm{Hgb})$ content of a serum sample flowing in microfluidic channels: resonator-microfluidic assembly (top) and resonance curve and fitted resonance frequency for different Hgb concentration. The $\mathrm{x}$-axis in the bottom right figure represents Hgb measurements by the gold standard Hgb technique (from [4]).
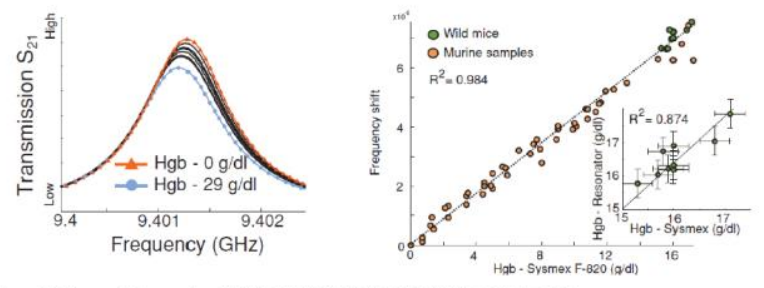

from T.H. Basey-Fisher et al., ADVANCED HEALTHCARE MATERIALS 3, 536, 2014

\subsection{Millimeter-wave photonic crystal resonators}

In spite of high Q-factors, the sensitivity limitation of WG resonators is determined by the character of the higher order mode, which implies large resonator dimensions and, consequently, moderate field enhancement. Moreover, since the microfluidic channel is arranged on top of the dielectric disc, the interaction volume comprises a relatively long section of the channel, making it unsuited for single cell detection. For frequencies above ca. $100 \mathrm{GHz}$, the low dielectric loss of high resistivity silicon (HRS) make it a more attractive resonator material, with the potential for integrated microfluidic channels. As an alternative to WGM resonators, defect modes in two dimensional photonic bandgap structures represent a promising alternative, and the micromachining capability of silicon enables microfabrication of complex structures by wafer scale processes. Fig. 2 from [11] shows our previous work on HRS 2D photonic bandgap defect resonators for dielectric measurement of liquids within a disposable glass capillary, which is inserted through a hole within the hexagonal photonic crystal lattice in close vicinity to the maxima of the resonant field. We have demonstrated highly accurate dielectric measurements on a variety of liquids, the actual interaction volume is only $4 \mathrm{nl}$. Apart from high sensitivity for small liquid volumes and high liquid-loaded Q-factors of several thousands the additional advantage of the use of millimeter-wave frequencies is the fact that the real part of the permittivity of water is only about $10 \mathrm{at} 100 \mathrm{GHz}$ rather than 70 at about $10 \mathrm{GHz}$ [13]. Since the walls of the microfluidic channels (glass, plastic) are of low permittivity, the electromagnetic fields are often expelled from the liquid due to its high permittivity. This leads to significant reduction of the electric field in Eq. 1 and to a flattening of the sensitivity of resonant parameters for the observation of small changes of the permittivity, for example due to cells or proteins (see Fig. 2). The advanced perturbation approach described in [11] takes this effect into account and enables quantitative dielectric measurements on nanolitre samples at $100 \mathrm{GHz}$ for the first time.

However, to achieve the ultimate goal of performing dielectric measurements on single cells of a few picolitres in volume, the sensitivity still needs to be improved dramatically. Microbeam resonators based on HRS represent a promising alternative for $\mathrm{mm}$ - and sub-mm wave frequencies for this purpose [12]. 

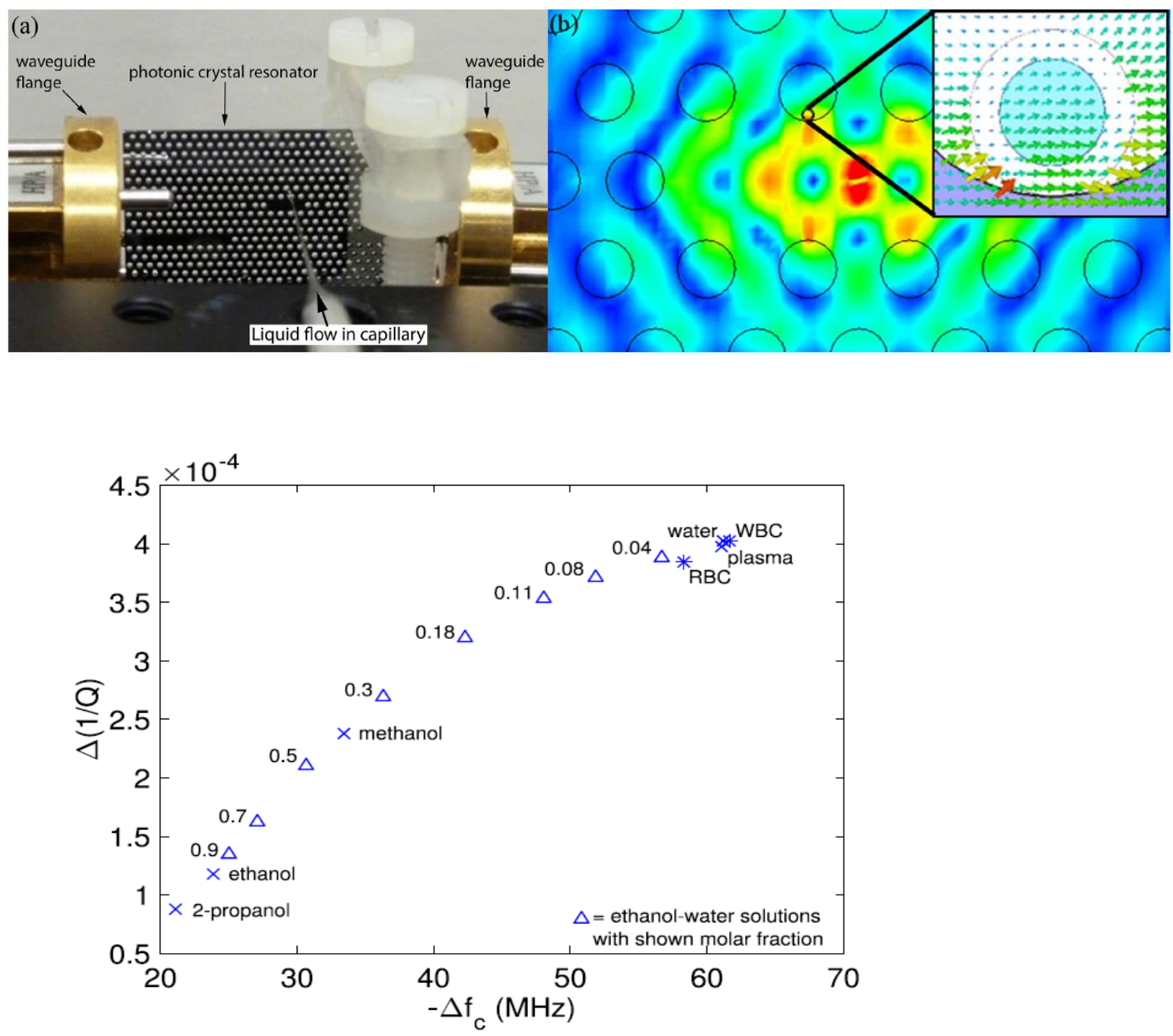

Fig. 2: $100 \mathrm{GHz}$ HRS photonic crystal resonator with a 100 micron thick quartz capillary in place for liquid measurements. The field simulations show the electric field distribution in the vicinity of the capillary. Results of $Q$ factor and resonant frequency measurements for a variety of liquids (from [11]).

\section{2: Coupled split ring - cavity resonators}

Only preliminary results for a frequency at approximately $10 \mathrm{GHz}$ are reported here, the full description of this novel approach will be the subject of a dedicated publication [14]. We have demonstrated that the advantages of metallic resonators (high electric field, small and controllable interaction volume) and the advantages of metal cavities or dielectric resonators (high Q-factors and high temperature stability) can be combined and even enhanced by the realization of a coupled resonator system with integrated microfluidics. To demonstrate the enhancement of the sensitivity possible with this concept, an equivalent circuit model has been developed (Fig. 3), which is composed of two RLC series resonant circuits with inter-resonator coupling by mutual inductance. Resonator 1 has a Q-factor of several thousands and represents a cavity type resonator to be used for measurement of liquid-induced changes of the resonant parameters. Resonator 2 represents a split ring resonator (SRR) with a microfluidic channel passing through both air 
gaps. As a first order approximation, we derived analytical expressions for the resonant frequency $\omega_{\mathrm{r}}$ and Q-factor of the SRR.

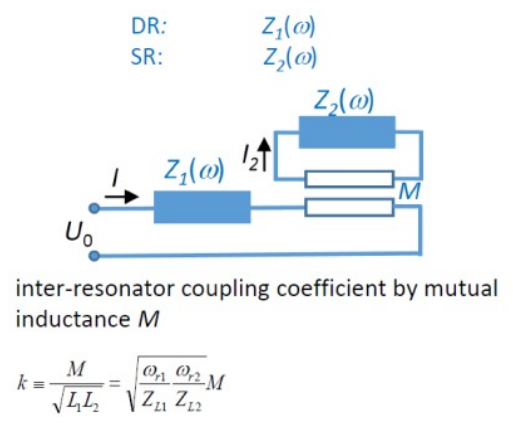

Fig. 3: Equivalent circuit of a coupled cavity (impedance $Z_{1}$ ) SRR resonator (impedance $Z_{2}$ ) system employing magnetic (mutual inductance) inter-resonator coupling.

$$
\begin{aligned}
& \frac{\omega_{r}}{\omega_{0}}=\sqrt{\frac{1+\kappa\left(\varepsilon^{\prime}-1\right)}{\left[1+\kappa\left(\varepsilon^{\prime}-1\right)\right]^{2}+\kappa^{2} \varepsilon^{\prime \prime 2}}} \\
& \frac{1}{Q_{d}}=\frac{\omega_{0}}{\omega_{r}} \frac{\kappa \varepsilon^{\prime \prime}}{\left[1+\kappa\left(\varepsilon^{\prime}-1\right)\right]^{2}+\kappa^{2} \varepsilon^{\prime \prime 2}}
\end{aligned}
$$

In Eq. 3a,b $\omega_{0}$ is the resonance frequency of the SRR without liquid, which may be taken from an eigenmode simulation. The filling factor $\kappa$ represents the electromagnetic filling factor of the microfluidic channel as a first order approximation, which neglects field expelling effects due to the high permittivity of water as discussed before. Note that the quality factor $Q_{\mathrm{d}}$ only includes dielectric losses due to the liquid and no Ohmic losses from the metal layer of which the SRR is made from. As confirmed by simulations, the Q-factor of the SRR with water filled channel is about 5 for our chosen geometry, which makes it nearly impossible to measure the resonant parameters directly.

Fig. 4 shows the results of the simulations qualitatively and explains the achieved amplification of sensitivity.

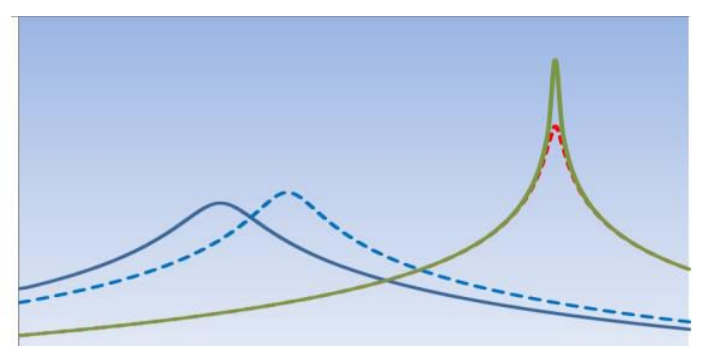

Fig. 4: Principal outcome of an equivalent circuit analysis for a coupled cavity-SRR system, illustrating the effect of inter-resonator coupling between a low Q-factor SRR resonance (blue) and a high Q-factor cavity resonance (green, red) (further explanation given in text).

Upon filling the channel with water, the resonance of the SRR (blue, full line) moves to the left side of the cavity resonance (green, solid line), typically 1-2 GHz below. However, the low Q-factor causes a significant overlap between the two resonances, which results in a reduction of the cavity Q-factor. For an optimized set-up the cavity Q-factor with water filled channel is about 2,000. A small change of the permittivity of the liquid under test, for example, due to a single cell passing through one of the SRR gaps, results in a slight increase of the SRR resonant frequency (blue, dotted line). This increases the coupling between the two resonators, resulting in a small Q-factor reduction for the cavity resonance (red, dotted line). The numerical analysis of the coupled resonator circuits shows that $1 \%$ change in liquid permittivity causes more than $2 \%$ of relative inverse $Q$ change according to the definition by Eq. 2 . This relative effect is 
of the same order as for a cavity which is completely filled with water, but at a Q-factor level of 1,000-2,000 which enables highly accurate detection of small changes in the resonant parameters.

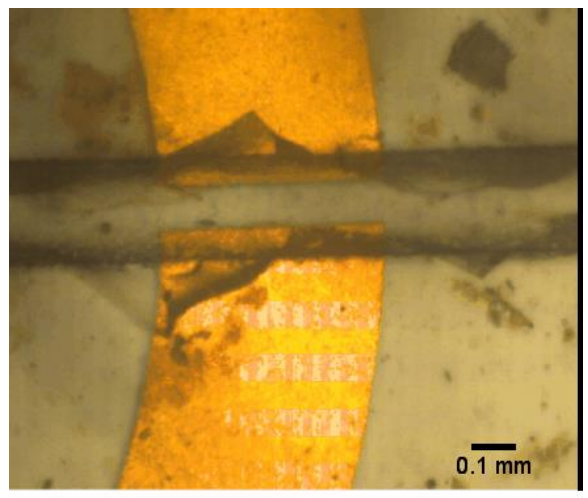

Fig. 5: Experimental demonstration of the response of a single polystyrene particle passing through one of the SRR gaps inside a water filled microfluidic channel. The particle causes a relative Qfactor change of the cavity resonance of about $2.5 \%$, which is easy to detect at a Q level of 1900 [14].

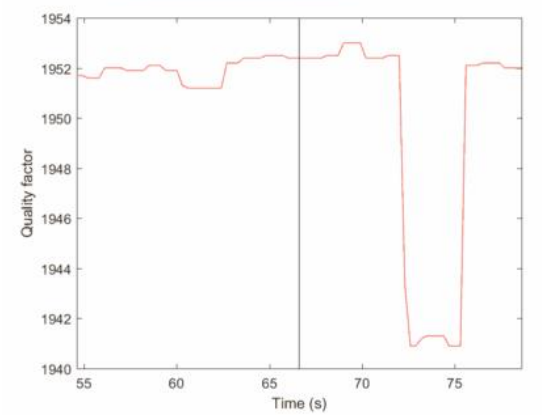

\section{Conclusion}

Resonator methods have been successfully employed for the dielectric measurement of small volumes of liquids within microfluidic channels, reservoirs and capillaries at micro- and millimeter-wave frequencies. Dielectric resonators and photonic bandgap resonators have great advantages over planar resonators due to their high Q-factors, which is important to achieve high sensitivity and fast measurement response. Coupled cavity-SRR systems at microwave frequencies have demonstrated extremely high sensitivity enabling single cell detection. Combining different frequency bands within one microfluidic device will be the way towards the most comprehensive characterization of single cells within a high throughput approach as an important step towards a liquid biopsy, enabling minimal invasive early state cancer detection and monitoring and personalized cancer therapy. In order to achieve this goal, multidisciplinary research and development approaches combining microwave, terahertz and photonic engineering with microfluidic technology and biomedical research are essential.

\section{Acknowledgment}

This work has been funded by UK's Engineering and Physical Science Research Council (EPSRC) within the priority area of "Healthcare Technologies" by the grants EP/M001121/1 and EP/M020398/1. 


\section{REFERENCES}

[1] Duffy MJ, "Tumor Markers in Clinical Practice: A Review Focusing on Common Solid Cancers", Med Princ Pract 22, 4-11 (2013)

[2] Alix-Panabieres C, Pantel K, "Challenges in circulating tumour cell research", Nature Reviews Cancer 14, 623-631 (2014)

[3] Adan A, Alizada G, Kiraz Y, Baran Y, Nalbant A, "Flow Cytometry: Basic Principles and Applications", Crit Rev Biotechnol. 14, 1-14 (2016)

[4] Basey-Fisher TH, Guerra N, Triulzi C, Gregory A, Hanham SM, Stevens MM, Maier SA, Klein N, "Microwaving Blood as a Non-Destructive Technique for Haemoglobin Measurements on Microlitre Samples", Advanced Healthcare Materials 3, 536-542 (2014)

[5] Basey-Fisher TH, Hanham SM, Andresen H, Maier SA, Stevens MM, Alford NM, Klein N, "Microwave Debye relaxation analysis of dissolved proteins: towards free-solution biosensing", Appl. Phys. Lett. 99, ISSN: 0003-6951 (2011)

[6] Chen LF, Ong CK, Neo CP, Varadan VV, and Varadan VK, "Microwave Electronics: Measurement and Materials Characterization", Chap. 6., John Wiley and Sons (2004)

[7] CST Microwave Studio, http://www.cst.com

[8] Chretiennot T, Dubuc D, Grenier K, "A Microwave and Microfluidic Planar Resonator for Efficient and Accurate Complex Permittivity Characterization of Aqueous Solutions", IEEE Transactions on Microwave Theory and Techniques 61, $972-978$ (2013)

[9] Abduljabar AA, Yang X, Barrow DA, Porch A, “ Modelling and Measurements of the Microwave Dielectric Properties of Microspheres" IEEE Transactions on Microwave Theory and Techniques 63, 4492-4500 (2015)

[10] Shaforost EN, Klein N, Vitusevich SA, Offenhaeusser A, Barannik AA, "Nanolitre liquid characterization by open whispering-gallery mode dielectric resonators at millimeter wave frequencies", J. Appl. Phys. 104, ISSN: 00218979 (2008)

[11] Hanham S. M., Watts C., Otter W. J., Lucyszyn S, Klein N. "Dielectric measurements of nanoliter liquids with a photonic crystal resonator at terahertz frequencies", Appl. Phys. Lett. 107, 032903 (2015)

[12] Hanham, S.M. et al., submitted for publication

[13] Markelz, A. G., "Terahertz dielectric sensitivity to biomolecular structure and function", Sel. Top. Quantum Electron. IEEE J. 14, 180-190 (2008).

[14] Watts C, Hanham SA, Ahmad MM, Klein N, oral presentation at the European Microwave Week, London Oct. 2016 